ENCYCLOPEDDIE Encyclopédie berbère

BERBERE

15 | 1995

15 | Daphnitae - Djado

\title{
Dieux africains et Dii Mauri
}

\section{G. Camps}

\section{OpenEdition}

Journals

Édition électronique

URL : http://journals.openedition.org/encyclopedieberbere/2258

DOI : 10.4000/encyclopedieberbere. 2258

ISSN : 2262-7197

\section{Éditeur}

Peeters Publishers

\section{Édition imprimée}

Date de publication : 1 avril 1995

Pagination : 2321-2340

ISBN : 2-85744-808-2

ISSN : 1015-7344

\section{Référence électronique}

G. Camps, « Dieux africains et Dii Mauri », Encyclopédie berbère [En ligne], 15 | 1995, document D48, mis en ligne le 01 juin 2011, consulté le 24 septembre 2020. URL : http://journals.openedition.org/ encyclopedieberbere/2258; DOI : https://doi.org/10.4000/encyclopedieberbere.2258

Ce document a été généré automatiquement le 24 septembre 2020

(c) Tous droits réservés 


\section{Dieux africains et Dii Mauri}

\section{G. Camps}

1 Dans un article de la Revue africaine paru en 1954 intitulé "L'inscription de Béja et le problème des Dii Mauri» il était établi une correspondance entre ces dieux et les nombreuses divinités locales que la terre africaine a nourries au cours de l'Antiquité et aboutissait aux conclusions suivantes: Il est des gentes (tribus) qui n'ont pas trouvé place dans la Romanitas, qui sont restées étrangères à l'intérieur même de l'Afrique romaine, elles se révoltent fréquemment et c'est en Maurétanie que ces révoltes sont les plus graves. On a pris l'habitude abusive mais assez logique d'appeler Maures les indigènes non soumis, quelle que soit la province qu'ils habitent.

2 Il est aussi des dieux et des génies indigènes, des divinités aux caractères fuyants qui n'ont pas trouvé place dans le panthéon romain. Ces dieux et ces génies attachés à telle tribu, à telle forme de relief ou à telle source restent donc farouchement berbères et africains. Pourquoi ne les appellerait-on pas du même nom que les indigènes non romanisés?

3 Cet ensemble, cette collectivité divine que les Romains veulent se concilier, ils lui donnent le nom de Dieux Maures puisque comme les Maures, ces dieux ne sont pas romanisés et restent « barbares ». Si on doutait encore de l'identité des Dieux Maures et des dieux locaux africains, l'inscription d'Henchir Ramdane permettrait d'apporter une conclusion formelle en faveur de cette assimilation puisqu'elle cite exceptionnellement le nom de trois Dii Mauri dont deux Macurtum et Varsis (sima) figurent également dans le panthéon local de Béja.

4 Les Dii Mauri ne retinrent plus guère l'attention jusqu'à la publication de la thèse de $\mathrm{M}$. Benabou en 1976 dont un des chapitres traitait à nouveau de la question. En une trentaine de pages, l'auteur présente plusieurs propositions. Tout en reconnaissant la possibilité de l'evocatio, M. Benabou insistait longuement sur une inscription de Micia (Dacie) dans laquelle des auxiliaires Maures dédicacent un temple à leurs Dii Patrii qu'il affirmait être les mêmes que les Dii Mauri des inscriptions africaines. Cette inscription lui permet de conduire un raisonnement particulier: reconnaissant, au culte des Dii Mauri un caractère principalement militaire, il se pose la question: «Est-ce suffisant pour affirmer avec G. Camps que ce culte est mêlé aux luttes contre les gentes non 
soumises? En fait il ne peut s'agir des Dieux des Maures non romanisés puisqu'ils sont invoqués comme dieux nationaux par les Maures de Micia au service de Rome " (M. Benabou, 1976, p. 317). Ce qui est, pour le moins, une pétition de principe car la dédicace est faite aux Dii Patrii et non aux Dii Mauri !

5 M. Benabou n'en reconnaît pas moins, lui aussi que le nom de Maure a reçu une acception très large ; il cite de nombreux exemples pris aussi bien chez les poètes que dans l'Hitoire Auguste mais il fait remarquer que ce qualificatif n'implique pas nécessairement un refus de la romanisation par ceux qui le portent ou le reçoivent. Cette opinion fut reprise par P.-A. Février, mais ni l'un ni l'autre de ces auteurs ne donne la moindre explication de l'extension démesurée de cet ethnique puisque au $\mathrm{VI}^{\mathrm{e}}$ siècle Procope citera des Barbares Maures au voisinage de Boréion, en Cyrénaïque (Aedil VI, 2, 21).

6 L'expression Dii Mauri a du naître, pense M. Benabou, dans les rangs de l'armée, elle a servi à désigner les divinités des auxiliaires maures. "Ce serait un moyen pratique de prendre possession de ces divinités, de les récupérer... sans pour autant leur faire perdre leur particularité " (M. Benabou, 1976, p. 325). Cette opinion reprend donc l'essentiel des conclusions de G. Camps et on constate la même concordance de vue sur la fréquence des dédicaces faites par des officiers ou des dignitaires. De même, $M$. Benabou admet les relations déjà proposées entre les dieux locaux et les Dii Mauri, mais selon lui ces derniers se situeraient à un échelon supérieur de la hiérarchie religieuse ; c'est oublier que plusieurs dieux locaux sont placés au même rang que les plus grands dieux de l'olympe.

7 «Les Dii Mauri, conclut M. Benabou, ont pu devenir une sorte d'abstraction à usage officiel ou privé permettant d'une part aux Romains de montrer leurs bonnes dispositions à l'égard des cultes africains et de leurs adeptes, d'autre part aux indigènes de faire preuve de leur aptitude à la romanisation. Ainsi après avoir rejeté les hypothèses antérieures, $\mathrm{M}$. Benabou revient à elles en les habillant d'une manière différente.

8 Dans une étude intitulée « Dii Mauri and Dii Patrii » parue en 1978, E. Fendress rappelle que la thèse de G. Camps fut discutée par M. Benabou qui suggérait que «les dédicaces aux Dii mauri doivent être étudiées dans le contexte particulier de l'armée d'Afrique qui comptait un grand nombre d'auxiliaires maures ». Ce à quoi on peut répondre qu'il y avait autant d'autres auxiliaires d'origine africaine: Numides, Musulames, Gétules, Afri... pourquoi ces dieux sont-ils qualifiés de «maures » sinon précisément parce que le terme «maure » avait reçu une acception particulière que l'hypothèse de G. Camps est pour le moment seule à expliquer. E. Fendress pense néanmoins qu'on peut pousser plus loin le raisonnement. Dans quelques inscriptions, la mention des Dii Mauri permet en quelque sorte de compléter l'universalité des divinités. Ces dédicaces apparaissent comme des synthèses de caractère social et politique. La réunion des dieux romains et africains sur la dédicace symbolise la paix retrouvée, leur mention apparaît comme un effort de conciliation entre les différents éléments de la société africaine.

9 En définitive les conclusions des études de M. Benabou et d'E. Fendress ne différent que fort peu de celles proposées vingt ans plus tôt. Aujourd'hui, l'identité des Dii Mauri et des dieux locaux sort renforcée d'un examen plus attentif des mentions littéraires ou épigraphiques de 50 divinités africaines et des 20 dédicaces aux Dii Mauri recensées jusqu'à ce jour. 


\section{Les Dieux africains locaux}

10 A un stade supérieur sinon de religiosité du moins de conceptualisation, le divin répandu dans la nature se personnalise. Des noms sont donnés à certaines entités qui font l'objet de dédicaces ou de citations qui nous ont été conservées. Parmi ces divinités quelques unes occupent une place prééminente, tel Saturne, dont l'omniprésence dans la province d'Afrique montre bien qu'il était vraiment le maître de ces terres et de leurs produits. Parmi les divinités secondaires, nombreuses sont celles qui gardèrent leur nom africain et se dérobèrent à toute assimilation à un dieu du panthéon gréco-romain. Certaines, cependant, sont regroupées sur les inscriptions, comme à Vaga (Béja) ou à Magifa (Ksar el-Boum), où elles constituent de véritables panthéons locaux voire régionaux. Mais la plupart nous apparaissent comme des divinités topiques, à peine distinguées des simples génies par le nom qui leur fut donné.

11 Afin de mieux saisir la complexité de ce cortège mythologique africain, il a semblé utile d'établir le catalogue complet de ces divinités.

12 Abbadir : à Manliana (Maurétanie césarienne)

1. - C.I.L. VIII, 21481 : ABBADIRI SA/NCTO CVLTO/RES IVNORES/ SVIS SVMITIS/

ARAM CONSTITV/PRO...

2. - Saint Augustin, Lettres, XVIII, 2 : «In numinibus Abbadires... »

En phénicien Abadir signifie "Père puissant » et aurait en particulier servi à désigner un bétyle. S'il en est ainsi la citation de saint Augustin permet de confirmer le maintien d'une certaine litholâtrie jusqu'au v $v^{\mathrm{e}}$ siècle. L'inscription de Manliana apporte la preuve de l'existence d'un culte organisé, mais Saturne, héritier de Baal Hammon, peut très bien se cacher sous cette appellation.

13 Aenon (ou Aeno) : Dieu et en même temps génie du municipe de Medda (Henchir Bed. Africa). L. Maurin et J. Peyras, «Uzalitana ». Les Cahiers de Tunisie, t. XIX, 1971, p. 71. A.E. $1973, n^{\circ} 602$ :

AENONI DE.. /GENIO MVNICI... /SAC.../

Ce dieu Aenon inconnu ailleurs, porte curieusement un nom de consonance grecque (cf. Aeneos: pérenne, éternel). Les inventeurs rejettent cette origine qui s'expliquerait difficilement. On ne voit pas à la suite de quelle circonstance un obscure municipe de l'Afrique intérieure aurait choisi un dieu grec, par ailleurs inconnu, comme génie municipal. On préfère y voir un nom libyque ou punique déformé.

Avlisva : Dieu connu par trois inscriptions de Maurétanie Césarienne occidentale, dans la région de Pomaria (Tlemcen) et par deux autels de Volubilis en Maurétanie Tingitane.

1. - C.I.L. VIII, 9906, à Pomaria : DEO/ SANCTO/AVLISVAE/ FL CASSI/ANVS PRAE/

FECT ALAE/ EXPLORA/TORVM POMARI/ENSIVM/ S...RIANAE

2. - C.I.L VIII, 9907, à Pomaria : DEO INVICTO / AVLISVAE/ M... /FL... /ALAE EXPLO PO/MAR GORDIA/ NAE ET PROC/ AVG N/

3. - C.I.L. VIII, 21704 à Ain Khial : DEO SANCTO AVLISVAE/ CALL VICTO.../CI E SIVLLC/... GENVO PIC./ POM ET.../... SARD.../

4. - M. Lenoir, «Inscriptions nouvelles de Volubilis». Bulletin dArchéologie marocaine, t. XVI, 1985-1986, p. 191-233 (inscription n ${ }^{\circ}$ 1) : AVLIS/ AVG /SACRV/

5. - M. Lenoir, Ibid. (inscription $n^{\circ} 2$ ) : DEO SANCTO AVLISVAE/VOTO DONVM DEDIT/VALERIVS VICTOR LIBER/TVS TVRNONIS SVTOR DED/XII K SEPT/ 
Le caractère africain de cette divinité paraît d'autant plus vraisemblable qu'on peut, avec $S$. Chaker, reconnaître dans son nom la racine berbère « $A W L$ » qui signifie « veiller sur ». Sur les deux inscriptions de Pomaria le dédicant est le préfet de l'aile stationnée à Pomaria et recrutée sur place. Il en est de même, semble-t-il, à Aïn Khial où il est fait également mention de la Cohorte II des Sardes dont le cantonnement se trouvait à Aitava. En revanche le dédicant de l'autel le mieux conservé de Volubilis est un affranchi, modeste cordonnier, qui dut faire un gros sacrifice pour la dédicace de cet autel dont l'une des faces porte un relief représentant Aulisua sous les traits de ConsusHercule, selon l'interprétation de $\mathrm{M}$. Lenoir qui considère Aulisua comme un dieu de la fertilité. Honoré à la fois à Pomaria et Aïn Khial en Césarienne et à Volubilis en Tingitane, et portant en ces trois lieux le même qualificatif de Sanctus, Aulisua n'est pas une simple divinité locale, mais un grand dieu maure. Peut-être faut-il l'associer à la gens des Bavares occidentaux, présente, comme lui, de part et d'autre des limites des deux Mauré-tanies.

16 Avzivs : C.l.L. VIII, 9014, à Auzia (Sour el-Ghozlan, ex Aumale, en Maurétanie Césarienne) : AVSio deo Genio et conservatori col/ eXtrictVs... /MAesia... / InSTITVAE... IVCVNDAE/ CONIVGI... HONORATAE... PRI/MOSAE HONORE... VS SVIS DE CLAVDIO IVVE/NALE PATRE ET DECENNIO VICTORINO FRATRE INSTAN/TE L CAECILIO

VICTORINO AMICO KAL IANVAR S PROV/ CLXXXV.

Cette dédicace datée de 225 s'adresse à un dieu local qui a donné son nom à la colonie d'Auzia et fut assimilé au génie de celle-ci. Il y a dans cette dédidace, comme dans la titulature de la divinité un intéressant mélange entre la conception libyenne d'un dieu local personnalisé et la notion toute romaine du génie tutélaire de la cité.

Bacax : C.I.L. VIII, 5504 à 05520 et 18828 à 018857 . cf J. et P. Alquier, Le Chettaba et les grottes à inscriptions latines du Chettaba et du Taya. Constantine, 1929, pp. 141-168.

Divinité honorée dans une grotte du Djebel Taya, au nord-ouest de Guelma, mais située en Numidie. Ce dieu est mentionné une soixantaine de fois dans les inscriptions qui tapissent les parois de la cavité. Ces inscriptions étaient gravées sur ordre des magistri de Thibilis qui venaient sur place chaque année, vraisemblablement au moment de leur entrée en fonction. La cérémonie avait généralement lieu le dernier jour de mars ou le premier jour d'avril. L'inscription la plus ancienne est de l'année 210, la plus récente de l'année 284.

Bacax est dit Auguste dans tous les textes conservés. La forme Bacques (C.I.L. VIII, 7420) et Bacquax (C.l.L. VIII, 20720) sont connues dans l'onomastique africaine.

Baldir, Baliddir. Dieu connu par trois inscriptions, deux de Sigus, une de Guelaat bou Sba.

1. - C.I.L. VIII, 5279 à Guelaat bou Sba (Africa proconsularis) : BALDIR AVG/ SACRVM/ MACEDO/PUB/ VOTVM SOLV/IT LI... AN.../

2. - C.I.L. VIII, 19121 à Sigus (Numidie) : DEO PATRIO/ BALIDDIRI AVG/SACRVM/ Q TARDIVS Q FIL / QVIRINA VICTOR/ STATVAM AEREM QVAM OB HONOREM FLAMONII DIVI SEVE/RI CASTELLI SIGVITANI/ POLLICITVS ERAT FAC/TVRVM SE EX X D LAMEI/ FICATA LIBERALITATE/ EX X MILLE CVM BASE/ TADII/ VICTOR IVNIOR ET/ SATVRNINVS ET HONORATA ET FE/LIX FILII ET HERE/DES EIVS DEDERVNT/ DEDICAVERVNT LDDD/

3. - C.IL. VIII, 19122 à Sigus (Numidie) : BALIDDIRIS AVG/ SANCTI PATRII DEI/ STATVAM/QVAM M. IVLIVS Q F PROCV/LVS OB HONOREM FL PERPETVI/DIVI MAGNI ANTONINI EX/SS II CC N SVMMAE HONORARI/AE EIVS HONORIS POLLICITVS/ET ADIECTIS AT/EA QVANTIT/TE EX SVA LIBERALITATE SS /ET 
AT BASEM SS CCC N EX/SS M DCN POSVIT IDEMQ/DEDICAVIT LDDD/

4. - Bir Eouel, région de Sigus (Numidie). J. Gascou et R. Guery ; « Inscriptions du Sud Constantinois ». Antiquités africaine, t. 25, 1989, p. 135-176 (p. 153) :

BALIDDIRI/ AVG SACRVM/ M. FABIVS F.../MVS ET/L.MAECIVS/EXTRICATV./V Q P S/ IDEMQ DEDIC/

La forme originelle du nom de cette divinité est incontestablement phénicienne, Ba'al 'Addir qui désignerait le Seigneur de la claie, c'est-à-dire de l'aire à battre (J. Ferron, REPPAL, III, 1987, p. 187-227). Un sanctuaire, élevé à Ba'al 'Addir à Bir Tlelsa, dans le Sahel tunisien, est mentionné dans une importante inscription néo-punique. A Cirta, de nombreuses stèles du sanctuaire d'EI Hofra sont dédiées à Ba'al 'Addir qui y possédait un temple (stèle $n^{\circ} 27$ ). La proximité de Cirta explique peut-être l'importance que Ba'al 'Addir conserva à Sigus, encore au $\mathrm{II}^{\mathrm{e}}$ siècle ap. J.-C, sous le nom contracté de Baliddir (Baldir à Guelaat bou Sba). Il est fort possible que le nom que porte alors la vielle divinité channanéenne (cf. Malk 'Addir de l'inscription d'Eschmunazar) ait été déformé par contamination du libyque. En berbère, en effet, il existe un verbe edder-idir qui signifie "vivre » et entre dans la composition d'anthroponymes, fait qui est constaté dès l'Antiquité. On peut citer, en particulier, le préfet de Castra Severiana qui portait le nom d'Ider (C.I.L. VIII, 9835). Ainsi les habitants de Sigus qui parlaient le libyque voyaient en Balidir un Dieu Vivant aux pouvoirs sans doute plus étendus que ceux du Maitre de l'aire à battre et donc des moissons, dénomination de la vieille divinité phénicienne. Il n'est pas impossible aussi que sous ces deux noms aient été révéré Saturne sous ses deux aspects de Frugifer et de maître du Temps.

Bonchor à Vaga (Béja, Africa proconsularis). A. Merlin. "Divinités indigènes sur un bas-relief romain de la Tunisie ». C.R.A.I.B.L., 1947, p. 355-371. A. E. 1948,nº 114 :

MACVRTAM MACVRGVM VIHINAM BONCHOR VARSISSIMA MATILAM IVNAM/ M AEMILIVS IANVARIVS ET Q AELIVS FELIX DE SVO FECERVNT ET DEDICRV/

Le bas-relief aux sept dieux de Béja

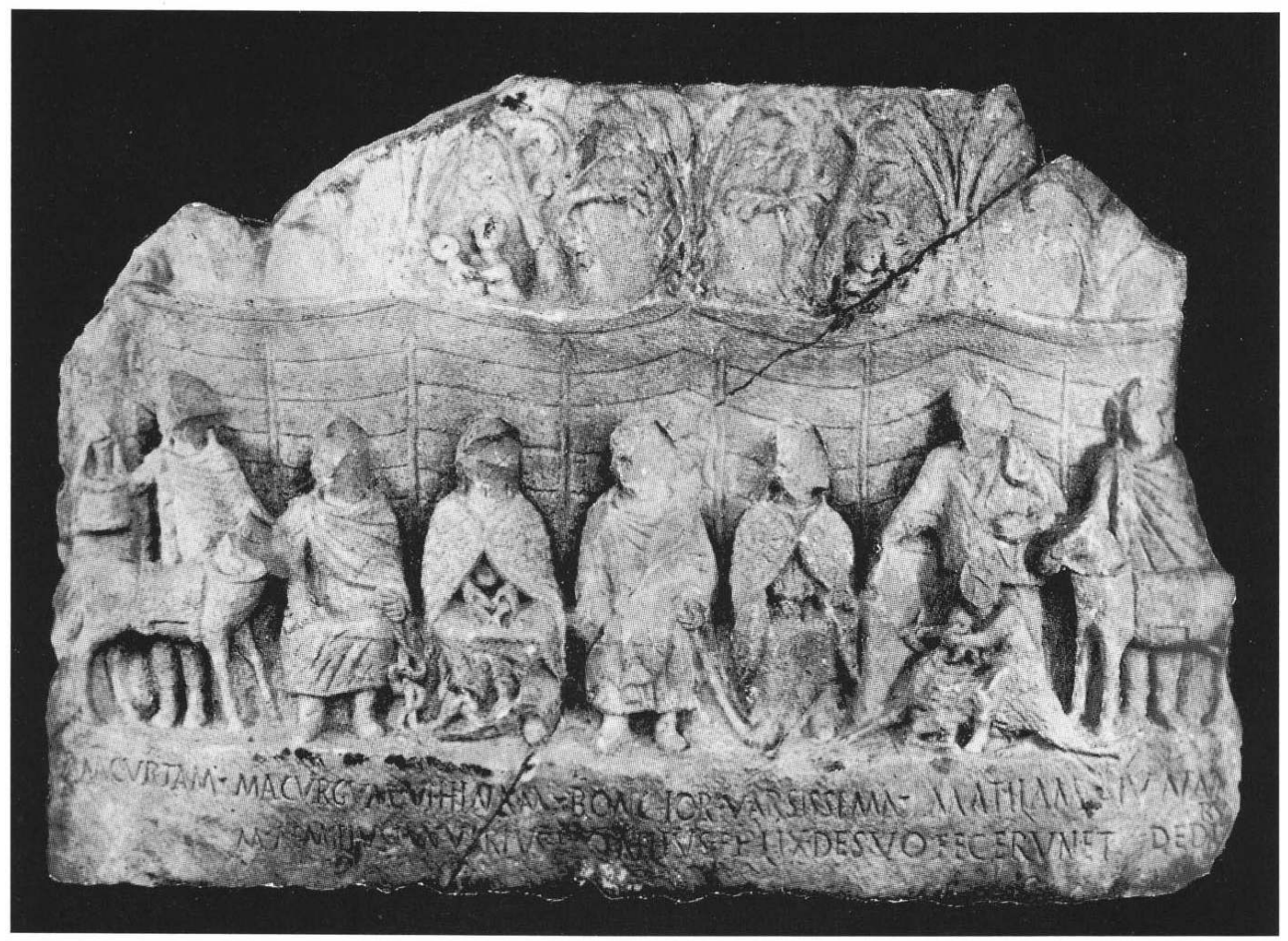



l'inscription. Bonchor occupe la position centrale, il est donc vraisemblablement la divinité la plus importante de ce panthéon local. Les sept dieux sont représentés de face, séparés d'un jardin par une tenture suspendue à des palmiers. Macurtam et Iunam sont des cavaliers, Bonchor drappé dans un lourd manteau tient dans sa main un gourdin, il est flanqué, à droite et à gauche de deux déesses, Vihinam et Varsissima revêtues, l'une et l'autre, d'une chape d'écaillés ou de plumes à moins que le sculpteur n'ait voulu figurer des mèches de laine. J.-G. Février voyait en Bonchor un nom punique qui serait la contraction de Bodmelqart («Serviteur de Melqart»), ce qui est douteux et même rejeté par M. Snycer. Il serait étonnant que le dieu principal de l'inscription de Béja porte un nom qui marquerait avec évidence sa subordination par rapport à un autre dieu d'origine phénicienne. Ce nom certainement théophore est très commun chez les Africains. Peut-être faut-il le rapprocher des Buccures cités par Arnobe comme divinités «maures»? Un autre bas relief découvert dans la voisinage de Béja et conservé au Musée du Bardo à Tunis représente également sept divinités en buste mais anonymes qui pourraient bien être les mêmes que celles figurées en compagnie de Bonchor. A Simithu (Chemtou) fut également découvert un bas-relief représentant huit bustes dont le quatrième est féminin; ils portent tous une chevelure abondante à mèches parallèles et sont revêtus d'une chlamyde retenue à l'épaule par une fibule circulaire. Un autre bas-relief similaire trouvé à proximité est plus fruste, la seule différence est que le quatrième personnage ne paraît pas féminin. Ces différents monuments, s'ajoutant aux inscriptions de Béja, d'Henchir Ramdan et de Magifa confirment l'existence de panthéons locaux ou régionaux.

Buccures: Arnobe, Adversus nationes, I, 36. Ces divinités ainsi que les Tisianes sont qualifiées de "mauri» par Arnobe qui est le seul à les citer. Ce nom rappelle celui du pharaon libyen de Tanis que les Grecs appelèrent Bocchoris; il peut être compris comme une forme plurielle de Bonchor (cf. supra).

Canapphari (datif) à Golas (Bu Njem*. Tripolitaine). R. Rebuffat, « Nouvelles recherches dans le sud de la Tripolitaine ».C.R.A.I.B.L., 1972, p. 319-339:

DEO MARTI CANAPPHARI AVGVSTO...

Dédicace du temple élevé par un détachement de la III Légion en 225 qui tient garnison à Golas sous le commandement du centurion T. Flavius Apronianus. Canapphare (ou Canappharis) est une divinité indigène assimilée au Mars romain. Elle présente donc les mêmes caractères que Sinifere, dieu de la guerre, cité dans ces mêmes régions trois siècles plus tard par Corripus. Il est probable que Sinifere et Canapphare soit le même nom transcrit laborieusement à des époques différentes.

Chalimace (datif) à Madauros (Africa proconsularis) LA. 2034 :

CHALIM/ACE AVG/SAC/VIRTIVS/ FLORENTI/VS VNA C/VM AVIS/ VSLA DD/

Autel de 0,55 m de hauteur. S. Gsell, qui publia le texte, hésite sur la seconde lettre de la deuxième ligne qui pourrait être un $\mathrm{G}$. Ce dieu n'est connu que par cette inscription. Au nominatif le nom devait être Chalimax; les désinences en ax sont fréquentes dans l'onomastique africaine (Baccax, Mazax...).

Cillenus à Thamugadi (Numidie). Ch. Vars. «Inscriptions découvertes à Timgad pendant l'année 1901 ». Rec. des Not. et Mém. de la Soc. archéol. de Constantine, t. 35, 1901, p. 218-274. A.E. 1902, nº 225 : CILLENO/PATRIO/DEO/PI... /

La qualité de Deus patrius est ambiguë; elle ne prouve pas nécessairement que la divinité est un dieu topique, comme l'affirmait un peu rapidement Vars. Il faudrait 
connaître l'origine du dédicant pour affirmer le caractère africain de ce dieu inconnu ailleurs. On ne pense pas qu'un rapprochement s'impose avec Silène ni avec Cillenius épithète de Mercure. On connaît, en revanche, la forme Cullen / Cullan/ Chullan dans l'onomastique africaine, chez Corripe et à Ghirza, dans l'inscription d'un mausolée.

Damio à Madauros (Africa proconsularis). I.A. 2036: DAMIONI AVG/ SACRUM/ C VIC/TOR CANISTR/THVG SPF/ID/

Le dédicant, le canistrarius C. Iulius Victor, est, semble-t-il, originaire de Thugga. Une dédicace à Ceres Maurusia est faite à Madaure par lulia Victoria qui est également canistraria. La qualité des dédicants, leur parenté, leur origine, permettent de penser que Damio est une divinité africaine, peut être associée à Ceres. Il paraît cependant difficile de retenir l'hypothèse de L. Joleaud qui rapprochait ce nom de celui que porte une variété de moutons sahariens à poils ras (daman).

Draco. Le culte du serpent est bien attesté en Afrique. C.I.L. VIII, 9326 à Caesarea (Maurétanie césarienne) : DEO MANUS DRACONIS M IVNIVS ASCLEPIADES VSLA

C.I.L. VIII, 15247 à Ain Guellaa, au sud de Thignica (Africa proconsularis) : DRACONI AVG SACRVM

C.I.L. VIII, 15378 à Numluli (Africa proconsularis) : DRACON. AVG. ACRV.

C.I.L. VIII, 17722 à Aquae Flavianae : NUMINI.MPHARVM ET DRACONI

I.A.II, 736 à CIRTA (Numidie) : D RAG AVG SAC. Un relief représente un serpent se dirigeant vers un autel.A ces inscriptions s'ajoute la mention du serpent de bronze à tête dorée que Salsa jeta à la mer, geste sacrilège qui fut la cause de son martyre.

Fudina. C.I.L. VII, 14444, à Henchir Ramdan (Africa proconsularis) :

(DIIS) MAVRIS/ FVDINA VACURTVM VARSIS/.

Au-dessus de l'inscription trois têtes sculptées représentent les trois divinités. Leur caractère indigène est indubitable. Cette dédicace permet certains rapprochements onomastiques avec les dieux de Béja qui doivent être considérés comme des dieux maures. Il est possible que Fudina qui, dans cette triade tient la même place que Vihinam à Vaga (voir infra) soit, comme celle-ci, une déesse de l'accouchement.

G.D.A. au Djebel Chettaba *, dépendant de Phua (Numidie). C.I.L VIII, 6267 à 6302 et 19249 à 1928. J. et P. Alquier. Le Chettaba et les grottes à inscriptions latines du Chettaba et du Taya. Constantine, 1929. pp. 169-183.

Rhar ez-Zemma est une grotte du Djebel Chettaba où était adorée une divinité qui n'est malheureusement connue que par ces initiales répétées dans une soixantaine d'inscriptions. Comme au Djebel Taya (Cf. Bacax) ce sont les magistri de la bourgade voisine, ici Phua, qui venaient en pèlerinage dans la grotte et y laissaient ces inscriptions en ex-voto. La seule différence vient de ce qu'à Phua un seul magister est mentionné lors de ces visites annuelles. On a voulu établir une correspondance entre le nom du Chettaba et celui du mont Giddaba que saint Augustin cite par trois fois, en particulier dans une des lettres récemment découvertes (Epist. 10*,6, 2). Mais ces textes laissent entendre que le mont Giddaba devait être proche d'Hippone ; aussi serait-ce particulièrement imprudent de tenter de développer l'énigmatique G.D.A. en Giddaba Deus Augustus; on pourrait tout autant développer en Genitor Deus Augustus, appellation qui s'applique à Saturne, ou Genius D... Augustus. Mais le parallèle qui s'impose avec la formulation abrégée B.A.S. (Bacax Augustus sacrum) fréquente au Djebel Taya, invite à penser que $G$ est l'initiale du nom de la divinité. 
31 Gurzil chez les Laguatan (Corippus V, 22-26). Au moment des combats les Laguatan lâchaient sur l'ennemi un taureau qui était censé représenter le dieu Gurzil dont on disait qu'il était né de l'accouplement du Dieu Ammon et d'une vache. D’après Corippe, les Laguatan possédaient des idoles en bois et métal de ce dieu. Il importe de noter que le nom de Gurzil se lit sur une inscription néopunique découverte à Lepcis Magna en 1846 ; or dans ce texte Gurzil précède Saturne, ce qui révèle son importance (F.A. Elmayer, Libyan studies, 13, 1982, p. 124). Ghirza, en Tripolitaine, a peut-être conservé le nom de ce dieu.

32 Haos à Civitas Popthensis (Ksiba, Africa proconsularis). C.I.L. VIII, 16750 :

HAOS AVG/SAC/L LEPIDIVS /PRIMVLVS SA/CERDOS HOC / LOCO INITIA/TVS ARAM/ POSVIT VO/TVM SOLVIT/DD

Cette divinité inconnue ailleurs est vraisemblablement un dieu topique, le fait que l'initiation ait eu lieu sur place renforce cette opinion. Ce nom paraît cependant peu africain et se rapproche de la forme dorienne du nom de l'Aurore. Une dédicace sous cette forme serait toutefois surprenante dans cette modeste bourgade africaine.

Iemsal à Thubusuptu (Tiklat, Maurétanie césarienne) C.l.L. VIII, 8834: IEMSALI/L PERCINIUS/L F STEL/ROGATVS/VSLA/

L'origine libyque du nom lemsal ( = Hiempsal) ne fait aucun doute. Ce nom était encore porté chez les Berbères au Moyen Age: le bisaïeul d'Ibn Toumert, le Mahdi des Almohades, se nommait Iamsal. Mommsen pensait que l'autel fut dédicacé non pas au roi Massyle mais plutôt à une divinité dont le roi portait le nom, on partage généralement cette opinion. On ne doit pas oublier cependant qu'une dédicace au roi Hiempsal a été trouvée à Thubursicu Numidarum (C.l.L. VIII, 17159) et qu'à Gadiaufala (Ksar Sbahi) une inscription en l'honneur du roi Guluga (= Gulussa) fils de Massinissa (C.I.L. VIII, 18752) confirme l'existence, à l'époque romaine, d'un culte royal dans le cœur de l'ancienne Numidie. En fait plusieurs éléments invitent à penser que les rois numides portaient des noms théophores, souvent sous une forme apocoristique, coutume qui facilita d'autant le développement d'un culte des souverains décédés.

Ieru à Guechguech (Numidie). C.I.L. VIII, 5673 :

IERV AVG SAC C IVL/ CRESCENS VIS / AB FECIT/

Cherbonneau (Rec. de la Soc archéol. de Constantine, t. 12, 1868, p. 393-456) avait proposé de lire IFRV en supposant qu'il s'agissait d'une divinité qui, comme Baccax ou G.D.A., était honorée dans une grotte (ifri en berbère), mais un réexamen de l'inscription et un estampage nous ont convaincu de l'exactitude de sa première lecture, celle retenue par le Corpus. Au-dessus de cette inscription rupestre une gravure représente un personnage dont la tête est radiée comme le serait celle d'une divinité astrale. Or le nom de Ieru correspond assez bien au nom donné, en berbère, à la lune (qui est dans cette langue du genre masculin) : Eior en tamachek, Iur en Zénatia de l'Ouarsenis, au Mzab et à Ouargla... Je ne pense pas que s'impose un rapprochement avec le nom donné à Dieu chez les Zagawa du Tchad oriental : Irou.

Iesdan (connu au génitif : Iesdanis) à Magifa (Ksar el-Boum, Africa proconsularis)

C.I.L. VIII, 16749 : DIIS MAGIFAE AVG Q T POLITICVS SIMVLACRA DEORVM N V/

MASIDENIS ET THILILUAE ET SUGGANIS ET IESDANIS ET MASIDDICE ET TEMPLVM /A FONDAMENTIS EX SVA PECVNIA FECIT EX SS VLIL N ITQ CAPITI VISO IPSIS ATPETENTIBVS CVM /SUIS OMNIBVS VSLA BBMB/

lesdan est l'une des cinq divinités de Magifa à qui Q. T. Politicus éleva un temple et cinq statues. Comme le note S. Gsell, la modicité de la somme (8 000 sesterces) convient plutôt à un édicule ou une chapelle d'autant plus que les simulacra des cinq dieux sont 
compris dans la dépense. Le caractère africain de lesdan et des quatre autres divinités ne fait aucun doute.

Ingirozoglezim à Vanisnesi (Hassnaoua, région de Bordj-bou-Arreridj, Maurétanie Sitifienne) C.l.L. VIII, 20627 : NVNDINA/ANNV QVOD/ PRAECEPIT/IOVIS ET IV/BA ET GENIVS VANISNESI/QVOD PRAECEPE/RUNT DII INGI/ROZOGLEZIM/

L'inscription de Vanisnesi place sous l'autorité de différents dieux la tenue d'une foire annuelle en ce secteur proche de la frontière de Numidie. Ces divinités sont Jupiter, Juba (non pas le roi mais la divinité dont les deux Juba ont porté le nom), le génie du lieu et les mystérieux Dii Ingirozoglezim dont la désinence semble correspondre à un pluriel, mais l'étrangeté du nom invite à la prudence. La lecture étant sûre, plusieurs auteurs se sont demandé s'il ne s'agissait pas d'une faute du lapicide ou de l'ordinator.

Iocolon à Naraggara (Sidi Youssef, Africa proconsularis). C.I.L. VIII, 16809 et LA. 1184 : IOCOLONI DE/O PATRIO M MEVIVS ROMANVS/ COMES AVG /N VIR EGREGIVS

Divinité inconnue en dehors de Naraggara. La qualité du dédicant pourrait faire douter $\mathrm{du}$ caractère africain de ce dieu, mais $\mathrm{M}$. Mevius Romanus semble bien originaire de Naraggara puisqu'un autre M. Mevius Romanus flamen perpetuum est enterré dans cette ville (LA. 1190). On a trouvé, voici une vingtaine d'années, à Césarée de Palestine, une inscription d'un centurion de la Legio VI Ferrata qui selon une proposition de H.G. Pflaum pourrait être le fils du flamen et le père du Comes. Ces trois personnages sont vraisemblablement parents avec les Maevii de Tébessa (LA. 3419). Mais ce gentilice est assez fréquent en Afrique on le connaît entre autres à Hadrumète, Uchi Maius, Thibilis, Cirta, Tiddis, Celtianis... Iocolon devrait donc être considéré comme une divinité indigène.

Iuba à Vanisnesi (Hassnouna Maurétanie sitifienne) voir Ingirozoglezim. C.I.L. VIII, 20627.

Il est remarquable que dans cette inscription, Iuba soit cité immédiatement après Jupiter. Est-ce le roi divinisé ? En faveur de cette hypothèse se trouve l'affirmation de Minucius Félix : «Iuba, Mauris voluntibus, deus est. (Octavianus, XXIII). Cependant on sait que les rois ont porté des noms théophores et Iuba me parait être une de ces divinités africaines auxquelles les rois et les chefs africains empruntaient leur nom. Dans les Bibans, au sud-ouest de Vanisnesi, on connaît, grâce à Ammien Marcellin (XXIX, 51, 44), la tribu des Iubaleni, dont le nom semble dériver de celui de Iuba. Au milieu du III siècle il est fait plusieurs fois mention d'un évêque nommé Iubaianus (Conciles de 255 et 256) on à peine à croire qu'ils doivent, les uns et les autres leur nom au roi Juba.

Irsiti (datif) à Aïn Regada (Numidie). C.I.L. VIII, 5667 : VOVTM QVEM/ PROMISIT HERCU/LI IRSITI ROGATVS /FECIT DEDICAVIT /LIBENS ANIMO/

A. Poulie qui publia cette inscription et décrivit le bas-relief qui l'accompagne, croyait que Irsiti est une corruption de l'adjectif hirsutus. Cette interprétation n'a pas été retenue, mais le caractère africain du nom Irsiti reste conjectural. La figuration d'Hercule brandissant la massue et portant la dépouille du lion de Némée est des plus classiques.

Ivnam à Vaga (Africa proconsularis). Voir supra Bonchor.

Un des sept dieux de Vaga. Il est figuré sous les traits d'un cavalier placé derrière sa monture à l'extrémité gauche de la scène, comme son parèdre Macurtam placé symétriquement à l'autre extrémité, il porte un manteau court retenu à l'épaule droite par une fibule. A. Merlin assimilait ces deux divinités équestres aux Dioscures*. A. Dunand, s'élevant contre cette interprétation jugée trop savante, se demandait s'il ne 
fallait pas lire simplement Lunam. Contre cette opinion on peut faire valoir que la forme accusative n'a pas de raison d'apparaître dans cette dédicace. De plus il s'agit d'un dieu et non d'une déesse, or chez les Grecs et les Latins la Lune, contrairement à ce qui se passe chez les Berbères, est bien du sexe féminin. Iunam doit donc être considéré comme une divinité africaine, équestre, de sexe masculin. Les Dioscures apparaissent souvent comme les assesseurs de Saturne et la découverte, à Musti, d'une dédicace aux Mauris Castoribus conforte la lecture proposée par A. Merlin.

Lilleo (datif) à Madauros (Africa proconsularis) C.I.L. VIII, 4673 et I.A 2053 :

LILLEO AVG/PRO SALVTE IMP CAE./M AVRELI SEVERI./LEX / C LICINIVS CRON/ CHRONA SACERD/ CAELT ARAS SVIS/SVMP FEC ET DED/

L. Joleaud a rapproché le nom de cette divinité de celui, peut être sous forme féminine, de Thililua, l'un des dieux de Magifa. On peut reconnaître dans ces deux noms, la racine berbère lilu qui s'applique à ce qui est brillant, propre ; le verbe ilil veut dire rincer aussi bien en Kabylie qu'au Mzab ou à Ouargla. Il n'est pas indifférent que cette dédicace à un petit dieu africain ait été faite par un sacerdos de Caelestis.

42 Macvrgvm à Vaga (Africa proconsularis) voir supra Bonchor.

Cette divinité porte un nom manifestement libyque, on reconnaît le trilitère MKR (mokran: grand) qui entre dans la composition de nombreux anthropony-mes. On connaît un Macargus à Golas (Bu Njem*) et un Magu(r)cum Fortunatus sur une stèle dédiée à Saturne à Henchir es-Srira. Ce serait de nouveaux exemples de noms théophores, toujours sous forme apocoristique chez les Africains. Macurgum, du moins à Vaga, semble jouer le rôle d'un dieu guérisseur. Il est représenté assis, tenant dans la main droite un volumen et de l'autre un bâton autour duquel s'enroule un serpent.

Macvrtam / V ( =M) acvrtvm voir supra Bonchor, à Vaga, et Fudina à Henchir Ramdan, inscription sur laquelle je propose de lire Macurtum au lieu de Vacurtum. Sur le basrelief de Vaga, Macurtam est avec Iunam l'une des deux divinités équestres. Dans la triade d'Henchir Ramdan, Macurtum occupe la place centrale qui était celle de Bonchor à Vaga, il est accompagné de deux déesses Fudina et Varsis(sima). Le nom de Macurtum apparaît dans plusieurs inscriptions libyques sous la forme MKRD.

Masgav (Masgava ?), au voisinage de Thugga (Dougga, Africa proconsularis), C.l.L. VIII, 27431:

MASGAV... / PRO SALVTE... /FELICI... / PVBLICOL... /I CPPP III M/ECACAIO/... /

Le nom de cette divinité est manifestement libyque. Un fils de Massinissa portait ce nom (Tite-Live, XLV, 13, 14). Aucune raison ne permet de penser que ce prince, qui ne laissa d'autre souvenir que cette seule citation, ait pu être divinisé et adoré encore plusieurs siècles après sa mort. Il s'agit, ici aussi, d'un dieu africain dont un prince massyle porta le nom. On connaît un Masgabes à Musculula (C.l.L. VIII, 27490), et un Masgivin préfet de Safar, dans la région d'Altava (C.l.L. VIII, 9835), ces noms sont peutêtre tirés de celui du dieu.

Masi... à Abizar (Kabylie, Maurétanie césarienne) C.l.L. VIII, 20731 :

TABLA DEO MASI..... On avait pensé qu'il pourrait s'agir de Massinissa divinisé, mais bien d'autres noms libyques commencent par ces mêmes lettres, parmi les noms de divinités connues on peut citer Masiden et Masidice à Magifa.

Masiden à Magifa (voir supra lesdan). Un des cinq dieux de Magifa. 

indifférent que Motmanius soit cité avant Mercure. Cette prééminence d'une divinité barbare sur un dieu latin paraît d'autant plus étonnante qu'elle émane d'un centurion. On doit remarquer que les dédicaces aux Dii mauri, particulièrement à Lambèse, sont souvent le fait d'officiers qui invoquent ainsi collectivement les dieux indigènes. En faveur de la qualité africaine de Motmanius on peut noter que le trilitère MTM entre dans la composition de nombreux noms libyques (MTMH, MTMHL, MTMRSH etc.) et le reconnaître, déformé, dans le nom de Metymannus (Pline l'Ancien, VII, 61) ou de Methymnus (Valère-Maxime, VIII, 13) que porte le plus jeune des fils de Massinissa et qui est connu aussi sous le nom de Stembanos (Polybe, XXXVI, 16, 12). 


$$
\text { C.I.L. VIII, } 14690 \text { : }
$$

MERCVRIO SOBRIO GENIO SESASE PANTHEO AVG SAC/PRO SALVTE IMP CAES M AURELI SEVERI ANTONINI AVG PIO FELICIS ET IVLIAE DOMNAE AVG MATRIS AVG ET CASTROR ET SENATUS ET/PATRIAE TOTIVSQVE DOMVS DIVINAE EORVM LVCILIA CALE FLAM COL THUB TEMPLVM A SOLO FECIT LIBENTIQVE ANIMO V / Les trois divinités possédaient chacune une niche dans le temple élevé par Lucilia Cale, flaminica de la colonie. Bien que simple génie, Sesas (ou Sesax) occupe la place d'honneur ; il semble bien être le génie local.

Divinité «maure » que le Mazax ( = Amazigh) assimile à Mars. Corripus le considère donc comme une divinité guerrière ; il s'agit très vraisemblablement du même dieu que les légionnaires de Golas (Bu Njem) vénéraient sous le nom de Mars Canapphari ( $c f$. supra). Des formes apparemment aussi éloignées sont révélatrices des difficultés que les Latins rencontrèrent lors de la transcription des noms berbères.

Suggan à Magifa (voir supra Iesdan).

C'est le troisième dieu cité sur l'inscription. Son nom, comme celui des autres divinités de Magifa, est typiquement africain. D'après S. Chaker (BCTH, nlle série, 19 B, 1985, p. 483-497), il pourrait signifier « être noir ». Ammien Marcellin (XXIV, 5, 21) cite, dans la région du Chélif en Maurétanie Césarienne, un chef mazice qui porte le même nom. Nouvelle preuve de la fréquence des noms théophores chez les anciens africains. Le nom est encore porté au Moyen Age, sous la forme Seggen (S. Chaker, ibidem, p. 491). Au nord de Ksar el Boum (Magifa) se dresse l'important relief du Djebel Doukkan, auparavant orthographié Souggan. Il n'est pas impossible que cette montagne ait conservé le nom de la divinité qui lui était peut-être associée. La Cosmographie d'Ethicus situe dans le sud de la Numidie un Mons Suggarem; on serait tenté de corriger en Sugganem.

Thililua à Magifa (voir supra lesdan)

On est tenté de voir en ce nom la forme féminine correspondant à Lilleo (voir supra)

ianes Arnobe, Adversus nationes I, 36. Ces divinités sont associées aux Buccures Mauri et méritent le même qualificatif. La correction proposée par plusieurs auteurs (Titanes au lieu de Tisianes) ne s'impose pas, elle est seulement plausible. En sa faveur on peut rappeler le caractère africain d'Antée et la place toujours tenue par les Géants dans le folklore nord-africain, mais ce thème mythique n'est pas particulier au monde berbère. La forme Tisianes a un aspect libyque par sa désinence «an» si fréquente en onomastique africaine.

Vacurtum (voir supra Macurtam)

61 Vanammon à Golas (Bu Njem, Tripolitaine). R. Rebuffat, Bu Njem, Encyclopédie berbère, XI, 1991, p. 1626-1642 (p. 1635)

Dédicace, faite par un notable de Golas, d'un édicule situé tout près du temple de Mars Canapphari. Le berbère invite à comprendre ce nom comme "Celui d'Ammon", il s'agirait donc d'une divinité secondaire faisant partie du cortège de Jupiter Ammon qui était honoré à Golas même où il possédait un temple.

Varriccala à Thabraca (Tabarka, Africa proconsularis) C.l.L. VIII, 17330 :

...PLVT VARICCALAE AVG/...VS ADVENTVS SACERDOS TEM/...SOLO SVIS SUMPTIB FECIT ET DEDIC/ Varricala est un qualificatif de Pluton et non une divinité distincte. J. Toutain trouvait à ce nom une origine punique (Baricgal). Mais une autre interprétation est suggérée par

Encyclopédie berbère, 15 | 1995 
la reconnaissance de la marque berbère de la négation "war" qui entre dans la formation de nombreux noms de personnes et de dieux.

Varsis à Henchir Ramdan (voir supra Fudina). Très vraisemblablement la même divinité que Varsissima de l'inscription de Vaga dont elle occupe la même place, à gauche du dieu principal.

Varsissima à Vaga (voir supra Bonchor). C'est une divinité féminine revêtue d'une chape d'écaillés ou de plumes. Elle ne possède aucun attribut. Son assimilation avec Varsis(sima) d'Henchir Ramdan permet de la compter parmi les Dii Mauri. Il est tentant de faire un rapprochement avec la Varsutina Maurorum de Tertullien.

Varsutina, Tertullien (Ad Nationes, 11, 8) cite Varsutina comme une divinité caractéristique des Maures, comme l'est Atargatis chez les Syriens et Caelestis chez les Afin. Je ne pense pas cependant qu'il faille restreindre le qualificatif maure à sa seule acception géographique et, de même que Caelestis était adorée en Maurétanie, Varsutina pouvait compter des fidèles dans les provinces d'Afrique et de Numidie, comme les nombreux autres Dii Mauri. On reconnaît dans ce nom, comme dans les trois précédents l'affixe «war» marque du négatif en berbère. Dans l'onomastique berbère "war» entre aussi bien en combinaison avec des substantifs qu'avec des formes verbales. Citons à titre d'exemple les noms de Verminad, fils de Syphax (Tite-Live), de Vertala, de l'inscription aurasienne de Masties, qui semble bien être le personnage que Procope nomme Ortalas (B.V., 11, 13, 19), de Vartilam à Guelma (Libyca, archéol. épigraph., t. 3, 1955, p. 202) de Varbas (I.L.A. 845), de Varubas (I.L.A.846), de Vartuminius (I.L.A. 1720) etc.

Vihinam à Vaga (voir supra Bonchor)

Le bas-relief qui surmonte l'inscription représente une divinité féminine qui comme Varsissima porte une chape d'écaillés ou de plumes; elle tient des ciseaux ou des forceps et un enfant est figuré à ses pieds. Merlin y voyait, avec beaucoup de vraisemblance, une déesse de l'enfantement.

Nous n'introduirons pas dans ce catalogue un prétendu Cereus dont le nom serait cité dans des cérémonies religieuses des militaires de Castellum Dimmidi (Messad) et de Golas (Bu Njem). Cereus ne serait pas, comme l'avait proposé G. Picard, une divinité archaïque associée à Ceres, mais simplement, selon R. Rebuffat, le cierge qui était allumé sur l'autel, lors des fêtes de Flore, le 3 mai (MEFRA, t. 94, 1982, p. 911-919). Doit disparaitre également des listes antérieur res des divinités africaines Tellus Gilva citée dans une inscription trouvée à Calama (C.I.L. VIII, 5305). Gilva n'est pas le nom d'une divinité locale associée à Tellus ou un qualificatif africain de cette déesse (cf. Pluto Varricala, Mars Canapphari, ou Hercules Irsiti) mais le nom d'une bourgade voisine de Calama, comme le révèle une des lettres récemment retrouvées de saint Augustin. Une ville de la région occidentale de la Mauritanie Césarienne portait le même nom. Cherbonneau a fait connaître une inscription d'Arsacal à CAVB AVG (39), le C.I.L. VIII, 6001, donne après relecture, G. AVB AVG, soit Genio Avb (...) Avg (vsto).

Ce catalogue des divinités africaines doit être, en revanche, complétée par une longue liste de génies topiques dont le caractère indigène n'est, toutefois, pas toujours assuré. Ils ont, en effet, très souvent un caractère officiel bien qu'étroite-ment rattaché au terroir. Nous citerons entre autres: Genius Subtarbati, à El Eulma, Genius Ausum à Sadouni, Genius Auburutensium à Guettar el Alech, Genius Thesecti (Henchir bou Skikine), Genius Municipi Satafis (Ain Kebira) qui sont des entités liées à des agglomérations; d'autres sont des maîtres inconnus des sommets: Genius Montis à Chemtou, Genius 
Montio Rufinae (?) à Khenchela, Genius Summus Thasuni (Aflou); d'autres sont des génies de fleuves: Genius Amsagae (Constan-tine), Genius Numinis Capitis Amsagae (Sila), Genius fluminis (Sig) etc.

En nous en tenant au catalogue des 50 dieux locaux retenus supra, il est possible de reconnaître plusieurs catégories ; émergent en premier lieu les dieux groupés par 7,5 ou 3 qui constituent de petits panthéons locaux. Tels sont les dieux de Béja et d'Henchir Ramdan dont les dédicaces sont assez proches et s'adressent à des divinités dont deux leur sont communes, du moins nous semble-t-il : Varsis/ Varsissima et Macurtam/ (M)acurtum. De même une des divinités de Magifa, Thililua, semble être connue sous une forme voisine (masculine?) à Madaure: Lileo (Lilleus?). Enfin l'inscription d'Henchir Ramdan nous apprend que ces dieux très nombreux sont aussi parfois évoqués collectivement sous l'appellation de Dii Mauri.

Une autre catégorie importante, non exclusive de la précédente, permet une approche différente ; elle comprend des dieux et déesses dont les noms sont également portés par des humains, soit que ces divinités aient été des hommes divinisés, soit, plus vraisemblablement, que les hommes aient porté des noms théophores. Entrent dans cette catégorie les noms de: Bacax, Bonchor, Iemsal, Iuba, Macurgum, Macurtam, Masgava, Matilam, Monna, Motmanius, Suggen.

Une troisième catégorie, elle aussi non exclusive des précédentes, comprend de rares noms de dieux qui se retrouvent dans la toponymie: Auzius, peut-être Masiddice (Vasidice $=$ Masidice), Suggen $(\mathrm{Dj}$. Souggan et Mons Suggaris ?).

71 Nous avons aussi des épithètes accolés au nom habituel d'une divinité du panthéon latin : Pluto Varricala, Mars Canapphari ( = Sinifer ?), Hercules Irsiti.

Il arrive que dans les dédicaces les dieux africains trouvent place à côté de divinités romaines ; ainsi Iuba est associé à Jupiter, Motmanius à Mercure, Sesas (ou Sesax), qui n'est qu'un génie, est mis sur le même plan que Mercure ou Panthée et occupe même la place d'honneur, au centre, dans le temple qui leur fut élevé. Dans une inscription néopunique de Lepcis Magna, Gurzil est cité avant Saturne.

Répartition des dédicaces aux dieux locaux et aux Dii Mauri

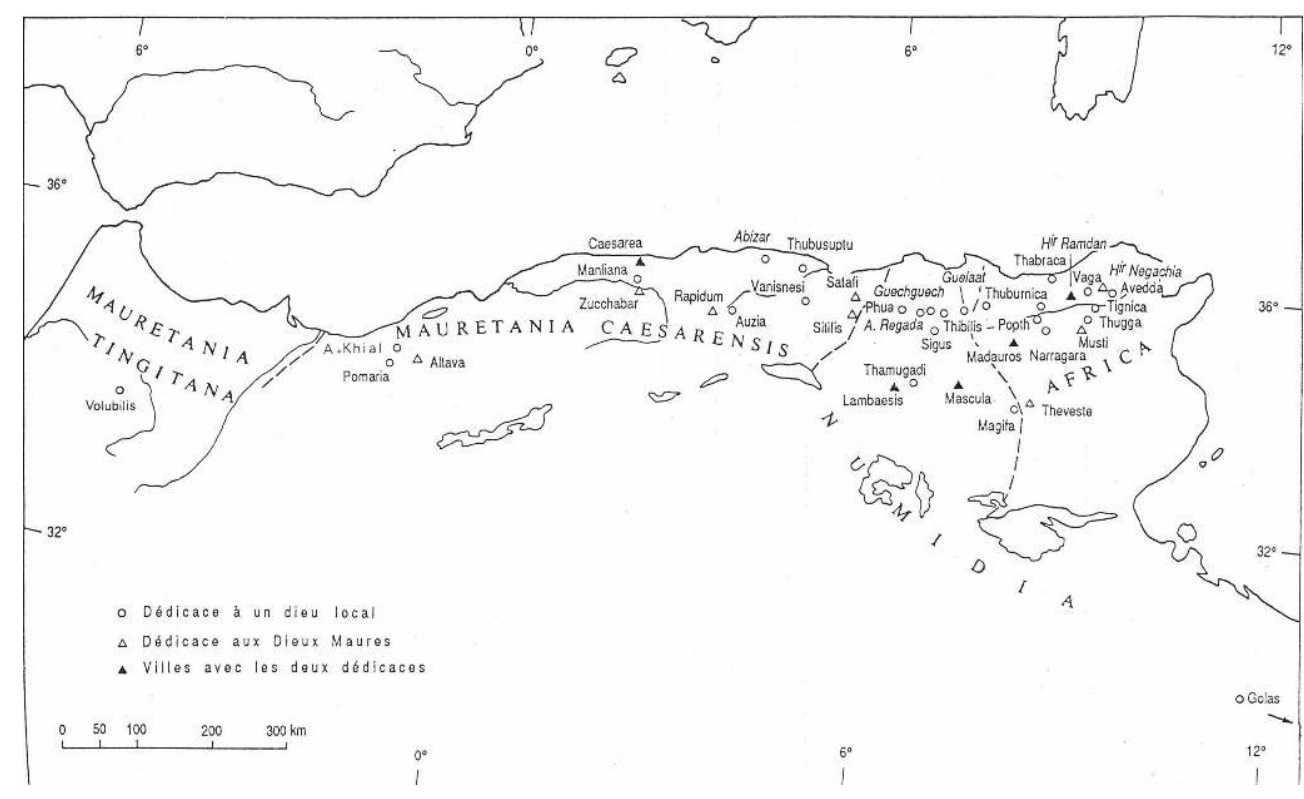



dehors de leurs provinces d'origine, sont citées par des auteurs chrétiens comme des dieux ou des déesses caractéristiques des Maures : tels sont les Tisianes et les Buccures Mauri d'Arnobe et la Varsutina Maurorum de Tertullien. Des dédicaces s'adressent de même à Diana Maurorum, à la Dea Maura, voire au Numen Maurorum.

a plupart des dieux africains que nous font connaitre les inscriptions ne sont que des noms, parfois réduits à des initiales qui conservent tout leur mystère (G.D.A. du Djebel Chettaba). Ces divinités ne règnent, pour la plupart, que sur des terroirs très restreints et se distinguent mal des simples génies locaux, ainsi Auzius est à la fois dieu et génie protecteur de la colonie d'Auzia.

\section{Les dédicaces aux Dii Mauri}

Cette foule de petits dieux jouissait auprès des Africains d'une ferveur certainement plus grande que le territoire sur lequel s'exerçait leur pouvoir et que nous laisse deviner un nombre infime d'inscriptions. Le plus grand nombre de leurs fidèles ne se souciaient pas de laisser un témoignage écrit de leur dévotion et n'en voyait certainement pas l'utilité. Cependant Romains et Africains latinisés, désireux de capter leur faveur, eurent recours à un procédé à la fois simpliste et efficace: ils les évoquèrent collectivement, écartant ainsi le danger qu'il y aurait à oublier l'une ou l'autre de ces divinités mal connues et vraisemblablement jalouses. Ils les appelèrent Dii Mauri.

Nous connaissons 20 dédicaces aux Dii Mauri auxquelles il est possible d'ajouter une demi-douzaine d'inscriptions s'adressant à des entités africaines telles que le Numen Maurorum, le Numen Mauretaniae, voire la Dea Maura; mais afin d'éviter toute confusion nous ne retiendrons que les mentions explicites des Dii Mauri. Voici donc le catalogue des 20 textes retenus, classés selon un ordre géographique, de l'ouest vers l'est, c'est-à-dire de la Maurétanie à l'Africa.

1. - ALTAVA: C.I.L. VIII, 21720 : DIIS MAVRIS/SALVTARIBVS/AVRELIVS E/ XORATVS DEC/ALAE PARTORVM/PRAEPOSITUS COHORTIS/SARDORVM SE/VERIANAE/

Sous Alexandre Sévère (227-235)2. - ALTAVA : G. Camps, «L'Inscription de Béja et le problème des Dii Mauri. » Rev. africaine, t. XCV111, 1954, p. 233-258 (p. 258). A.E, 1956, $\mathrm{n}^{\circ} 159$ :

DIIS PROSPERIS/MAVRIS SALVTA/RIBVS C FAN/NIVS IVLIANVS/A MILITIIS PRAEF/ COHORTIS SARDO/RVM V S L A/

3. - CAESAREA : B.C.T.H.S., 1920 , p. LX. A.E. $1920, \mathrm{n}^{\circ} 31$ :

MAVRICIS/C STERTINII/VS AEMILIA/NVS PROC AVG/

Le même texte est gravé sur l'autre face de l'autel.4. - CAESAREA : C.l.L. VIII, 9327 : DIIS MAVRICIS/ M POMPONIVS VL/TEL-LIANVS TRIBVS/ MTLITTIS PERFVNCTVS/ PROC AVG AD CVRAM/ GENTIVM PRAEF CLAS/ SIS GERMANAE/

Probablement du III siècle.5. - ZUCCHABAR : C.l.L. VIII 21486 : DIIS PATRIIS ET MAVRIS/ CONSERVATO-RIBVS/ AELIVS AELIANVS V P/PRAESES PROVINVCIAE/ MAVRETANIAE CAES/ OB PROSTRATAM GENTEM/BAVARVM MESEGNEITSIVM/ PRAEDASQVE OMNES AC FAMI/LIAS EORVM ABDUCTAS/ VOTVM SOLVIT/

Aelius Aelianus fut praeses de Maurétanie césarienne dans la seconde moitié du $\mathrm{III}^{\mathrm{e}}$ siècle. L'inscription daterait de 284-288. J. Carcopino (BCTH, 1920, p. CV) propose de lire 
Bavarum Mesgnensium.6.-RAPIDUM : C.l.L. VIII, 9195 : (d)IIS DEABVSQVE CONSECRATIS V..../ NVMINI IOV. SILVAN./MERCVRIO FORTVNAE/VICTORIAE CAESSS/ DIS MAVRIS / M FVRNIVS DONATVS EQ. FL PP/EX PRAEF G MASAT OR I /CVM SVIS FECIT ET IT/

La mention caesss est peu sûre en raison soit d'un martelage soit d'une érosion de la pierre. M. Le Glay suggère de lire Caeles(tis). Cette lecture présente quelques difficultés : Caelestis serait la seule divinité dont le nom aurait été abrégé sur cette inscription; de plus la place qu'elle occuperait dans le texte épigraphique, après Victoria et avant les Dii Mauri, me paraît en contradiction avec son rôle éminent dans le panthéon africain. Si on retient CAESSS avec martelage, l'inscription pourrait dater des règnes de Valérien, Gallien et Valérien le Jeune (255-259). On retiendra que le dédicant est un ancien praefectus gentis.7. - SITIFIS : C.I.L. VIII, 8435 :... OLL DID/PATRIS ET HOSPI/TIBVS DUS MAURI/

CIS ET GENIO LOCIS/M CORNELIVS OCTAVI/

M. Cornélius Octavianus fut praeses de Maurétanie césarienne entre 255 et 2588. SATAFIS : C.I.L VIII, 20251 : DIIS MAVRIS...SER/VATORIBVS ET GENIIS/

SATAFIS SALLVSTIVS EX QUAESTIONARIO /TEMPLVM DE SVO /EXORNAVIT/VSLA/

Le municipe de Satafis (Aïn Kebira) qui possédait donc un temple des Dii Mauri avait également consacré un lieu de culte au Numen Maurorum (C.I.L. VIII, 20252).9. LAMBAESIS : C.I.L VIII, 2637 : PRO SALVTE/ IMP ANTONI/NI AVG PII/ ET SENATI P R /ET FVSCINI LEG/ C V ET LEG LIL / AVG ET AVXL/LIS EIVS. /ATIVS S /RD M AVRI S /

Fuscinus fut légat en 157-159; cette inscription serait la plus ancienne évoquant les Dieux Maures, mais malheureusement la lecture [Mau]ris est conjecturale.10. LAMBAESIS : C.I.L. VIII, 2638 : PRO SALVTE DN/ SEVERI ALEXAN/DRI PII

FELICI AVG/ DIS MAVRIS M PORCIVS EA/SVCTAN 7 LEG/XX VAL V SEVERAE/VSLA/

Cette inscription est postérieure à 222, année de l'avènement d'Alexandre Sévère et moment où le centurion $M$. Porcius Iasuctan (lecture plus sûre que Easuctan) commande la garnison de Golas (Bu Njem, Tripolitaine). Le nom orthographié tantôt Iasuctan, tantôt Iasuchtan, est connu dans l'onomastique libyque sous la forme YSKTN (R.I.L. 241, 256) ; ce même nom figure à Maktar dans la liste des Juvenes, et sur une inscription néo-punique. A Bu Njem même, un ostracon mentionne un chamelier qui se nomme aussi Ias-suchtan.11. - LAMBAESIS : C.I.L. VIII2639: DIS MAVRIS/ AVG SACR/ L FLAVIVS / M FIL

QVIR/GEMINVS/KALAMA MIL LEG III AVG/VSTA/Cette inscription est certainement antérieure à 237 puisque la mention de la III légion a été martelée. L'indication de la tribu Quirina et les tria nomina invitent à dater cette inscription du début du $\mathrm{III}^{\mathrm{e}}$ siècle au plus tard.12. -

LAMBAESIS : C.I.L. VIII 2641 : MAVRIS BARBA/RIS SERVILIVS IMPE/

TRATVS VO SO LIB AN/ VICTOR VENI VIC ME FAC/

13. - LAMBAESIS : C.I.L. VIII, 2640 : DIS M AU/RI S SAC/ L PVRTISI/VS FIRMVS/ HAST/ CVM POPILIA MAR/CIANE CON/JVGE FECER/ LA/

Certainement postérieure à Septime Sévère puisque le hastatus L. Purtisius Firmus a une femme légitime (conjux)14. -LAMBAESIS : B.C. T.H.S., 1968, p. 221 : MAVRIS/AVG/ L CAECILIVS FELIX / VSLA/

15. - MASCULA : Bull. mens, de la Soc. archéol. de Constantine, $\mathrm{n}^{\circ}$ 63, 1933, p. 210 :

DIIS MAV/RIS AVG/VERNA AVG /NN VERNA EX/ACTOR REG MASC TEMPLVM / CONLAPSVM/ A SOLO SVIS/ SVMPTIBVS/ FECIT DD/

16. - MADAUROS : LA. 2078 : SAEPE SACRVM SANCTIS MAVRIS FACIAS LIBENS. Table pouvant se lire dans plusieurs sens, sur le forum même de Madaure.17. - THEVESTE : I.A. 3000 : 
DIBVS MAVRIS IVL DO/NATIANVS SACERDOS/DAE CAELESTIS AVGVSTAE/ EX VISO SVO CVM SVIS OMNI/ BVS SOLVIT S/

18. - HENCHIR RAMDAN C.I.L. VIII, 14444 : DIIS MAVRIS /FVDINA VACVRTVM VARSIS/

Cette inscription apporte la preuve que les dieux locaux sont identiques aux Dii Mauri.Vacurtum doit très vraisemblablement se lire Macurtum (cf supra).19. - MUSTI A.

E. 1968, nº 590 : D IIS MAVRIS CASTORIBVS AVGG SAC/ PRO

SALVTE IMP CAES L SEPTTMI SEVERI PII PERTINACIS PP ET IMP CAES M AVRELII/ (ANTONL)NI PII FELICIS ET PVBLII SEPTIMI GETAE NOB CAES AVG G ET IVLIAE DOMNAE / ET FVLVIAE PLAVTILLAE AVGG P...PERCELLIVS SATVRNINVS II VIR P F ET DEDIC/

Cette dédicace date des années 203-204. La qualification de Mauri donnée aux Castores, au cœur de la Byzacène et fort loin de la Maurétanie, explique, dans un certain sens, la présence des deux divinités équestres Macurtum et Iunam sur le bas-relief de Béja. Macurtum et Iunam sont les Castores africains, maures.20. - HENCHIR NEGACHIA : C.1.L. VIII, 14438 et A.E. 1956, n 158 :....AVG CER ER I B...AVG/ M AURIS AVG..../.o PVBLICO ORDINIS CONCESSVM MVLTI

L'état de l'inscription retrouvée par J. Peyras ne permet ni de confirmer, ni d'infirmer, la lecture de Mommsen présentée dans le Corpus ; elle paraît vraisemblable.

Quelles divinités se cachent sous l'appellation collective de Dii Mauri? L'inscription de Rapidum ( ${ }^{\circ} 6$ de notre catalogue) montre qu'il ne s'agit ni de Jupiter, ni de Mercure, ni de Saturne, ni de la Fortune ; des divinités féminines, parmi ces Dii Mauri, ne peuvent non plus être confondues avec Caelestis puisque la dédicace de Théveste $\left(n^{\circ} 17\right)$ est faite par une sacerdos de cette déesse. En fait ces divinités ne sont pas assimilables aux divinités majeures de l'Olympe. En revanche, l'inscription d'Henchir Ramdan ( $\left.n^{\circ} 18\right)$ donne sans conteste le nom de trois de ces Dii Mauri : Fudina, Vacurtum ( = Macurtum), Varsis(sima), dont deux sont également nommés et figurés sur le bas-relief de Béja. On sait que sur ce même bas-relief deux dieux cavaliers Macurtam et Iunam ont été assimilés aux Dioscures, or la dédicace de Musti, qui date des premières années du III siècle, s'adresse précisément aux Castores qui sont qualifiés de Mauri. La même inscription de Béja et le bas-relief qu'elle commente accordent la place d'honneur, dans ce panthéon local, à Bonchor, dont la forme plurielle, qui ne peut être que Bonchores, rappelle les Buccures Mauri d'Arnobe. Un rapprochement aussi tentant s'offre à l'imagination entre Varsissima et Varsutina Maurorum citée par Tertullien. Certes ces approximations peuvent paraître trop faciles et rendre bien fragile notre argumentation, mais quand on prend la peine d'examiner les difficultés que les Latins ont eu à transcrire les noms berbères, de tels rapprochements paraissent moins risqués. N'ont-ils pas écorché Makaoucen (MKWSN) en Micipsa, rajouté un 1 final au nom de Mastanaba et nommé tantôt Canapphar (ou Canappharis) tantôt Sinifer le Mars libyen?

Des comparaisons, certes imprudentes, que nous établissons entre inscriptions et citations littéraires, il résulte que les dieux de Béja, d'une manière ou d'une autre, peuvent être qualifiés de Mauri, comme ceux d'Henchir Ramdan et les Castores de Musti.

Les Dii Mauri sont bien les mêmes dieux africains dont certaines inscriptions nous donnent les appellations locales. Il est donc intéressant de comparer les deux séries de dédicaces qui nous ont été conservées : celles aux dieux locaux nommément désignés, celles aux Dii Mauri invoqués collectivement. 
81 Les qualificatifs donnés aux dieux locaux n'apportent pas d'éléments à une meilleure connaissance de ces entités. Ils ne font que révéler une vénération banale : ils sont dits Augustus (11 fois), Sanctus (4 fois), Patrius (3 fois), ce qui confirme leur caractère local ; un seul dieu, Aulisua, invoqué il est vrai par des militaires, est dit Invictus.

Les Dii Mauri (appelés parfois Maurici) sont souvent invoqués sous cette seule qualification (9 fois), ils sont aussi Augusti (5 fois), Patrii (2 fois), Sancti (1 fois), Immortales (1 fois), ce qui ne nous apprend pas grand chose, mais ils sont aussi Salutares, Conservatores, Prosperi, Hospites, qualificatifs qui insistent sur leur caractère bienveillant et protecteur. Dans une dédicace ils sont dits Barbari $\left(n^{\circ} 13\right)$, ce qui fait presque pléonasme et montre bien que celui qui s'adresse à eux les sent étrangers à son univers culturel.

83 Les différences de qualificatifs donnés aux dieux locaux et aux dieux maures sont cependant révélatrices, non pas d'une différence de nature entre ces deux groupes de divinités, mais de l'état d'esprit différent des auteurs de ces dédicaces. Dieux locaux nommés individuellement et Dii Mauri confondus, le plus souvent, dans la même invocation, n'ont pas la même clientèle.

En recensant les renseignements sur la qualité des dédicants, il apparaît que le culte des dieux locaux est plus «populaire» que celui des Dii Mauri. Ainsi parmi ceux qui invoquent les premiers $46 \%$ mentionnent leur tria nomina, pourcentage qui s'élève à $77 \%$ dans le second cas, or les rares éléments chronologiques données par les inscriptions permettent de confirmer la contemporanéité de ces différentes dédicaces qui occupent la fin du $\mathrm{II}^{\mathrm{e}}$ siècle et toute la durée du $\mathrm{III}^{\mathrm{e}}$ siècle.

Bien plus significatif est l'examen comparatif des fonctions et des qualités des dédicants. On peut, pour simplifier, établir six catégories parmi eux. Sont dits « simples particuliers" les dédicants qui se nomment sans mentionner leur fonction. Les " magistrats municipaux » duumvir, édile, magister, constituent la seconde catégorie. La troisième est réservée aux personnes qui font état de leur fonction ou de leur qualité dans le domaine religieux : flamen, sacerdos, canistrarius, cultores. Viennent ensuite les fonctionnaires impériaux exerçant une procuratèle: procurator, praefectus gentis, jusqu'au simple exactor, qui constituent la quatrième catégorie. Sont rangés sous la rubrique "militaires", soldats et officiers subalternes jusqu'au grade de centurion. Enfin Praeses, Legati, Comités Augusti, Duces, tous personnages de haut rang constituent la dernière catégorie.

Le tableau comparatif de ces différentes catégories sociales est particulièrement suggestif.

\begin{tabular}{|c|c|c|}
\hline DEDICANTS & DIEUX LOCAUX & DII MAURI \\
\hline Simples particuliers & $55,0 \%$ & $6,25 \%$ \\
\hline Magistrats municipaux & $15,0 \%$ & $18,75 \%$ \\
\hline Flamines, Sacerdotes, etc. & $12,5 \%)$ & $6,25 \%$ \\
\hline Fonctionnaires impériaux & $2,5 \%$ & $18,75 \%)$ \\
\hline Militaires & $12,5 \%$ & $43,75 \%\} 81,25 \%$ \\
\hline Personnages de haut rang & $2,5 \%$ & $18,75 \%$ \\
\hline
\end{tabular}

Ainsi ce simple examen montre que le culte des dieux locaux est surtout affaire de «civils» (82,5\%), tandis que ceux qui invoquent les Dii Mauri sont surtout des 
«militaires », des fonctionnaires impériaux, des gouverneurs (81,25\%), personnages qui doivent traiter des "affaires indigènes", gentes non encore romanisées qu'il importe de gouverner $\left(n^{\circ} 4\right)$, de maintenir, de contrôler $\left(n^{\circ} 6\right)$, voire de combattre $\left(n^{\circ} 5\right)$. Contrairement à l'opinion ancienne de J. Toutain, en partie reprise par E. Fentress, qui voyait dans le culte des Dieux Maures la manifestation d'un patriotisme maure et aux conclusions ambiguës de $M$. Benabou, ce culte a un caractère essentiellement officiel et militaire en relation étroite avec le maintien, dans les provinces africaines, de gentes demeurées « barbares », pour ne pas dire berbères.

Il n'est pas indifférent que, malgré leur nom, ces divinités soient évoquées autant dans les autres provinces africaines qu'en Maurétanie. Leurs autels sont particulièrement nombreux à Lambèse, siège de la Légion (6 sur un total de 20 !). On en connaît un autre dans une ville de Numidie (Mascula) et 5 dans des villes de Proconsulaire (Theveste, Madaure, Henchi Ramdan, Henchir Negachia et Musti). En Maurétanie Césarienne, les dédicaces aux Dii Mauri les plus occidentales sont celles d'Altava. Caesarea, Zucchabar, Rapidum et la région de Satafis sont les autres localisations.

Jusqu'à présent aucune inscription aux Dii Mauri n'a été découverte en Maurétanie Tingitane, la plus « maure » cependant des provinces africaines puisque les Maures en sont originaires.

89 Le qualificatif de Maurus n'est donc pas lié au découpage provincial de l'Afrique romaine. On sait, en effet, que le nom des Maures déborda rapidement les limites administratives de la province double issue de l'intégration à l'empire de l'ancien royaume de Juba II et de Ptolémée. En fait, au cours de la domination romaine, on assista à la disparition progressive mais rapide, du nom des Numides (sauf pour la tribu regroupée autour de Thubursicu dit Numidarum, une fraction à Assuras et un troisième groupe aux environs de Sertei, dans la Medjana, donc en Maurétanie) et son remplacement par celui des Maures pour qualifier les Africains non romanisés, ceux que saint Augustin (Epistol. 199) nommait les "gentes barbarae». Ce glissement onomastique dépassa les limites de l'Africa, dès l'Antiquité tardive: nous avons vu qu'au vi siècle, Procope situe des «Barbares Maures" au voisinage de Boreion, en Cyrénaïque; au Moyen Age le terme maure (moro) sert à désigner, pour les Espagnols, d'abord les Africains musulmans qui effectuèrent la conquête " arabe ", puis n'importe quel musulman ; c'est ainsi que l'on qualifie aujourd'hui de " Moros » les Philippins de religion islamique. Pour en revenir à l'Afrique romaine, les populations de la partie orientale: Numidae et Afri furent en grande partie assimilés, romanisés alors que les Maures (dont ceux de Césarienne descendaient en fait des Numides Masaesyles) conservaient, pour la plupart, leurs structures sociales et politiques. Au $\mathrm{III}^{\mathrm{e}}$ siècle, époque du plus grand nombre de nos inscriptions, ils constituent encore de puissantes tribus conduites par des principes, voire des rois, tels les Baquates* en Tingitane, les Bavares* en Césarienne, ou de grandes confédérations organisées, comme aujourd'hui les Ayt 'Atta*, suivant une segmentarité quinaire, tels les Quinquegentanei* de Kabylie contre lesquels combattit l'empereur Maximien.

Il n'est donc pas surprenant que "maure " ait, dans l'Afrique romaine, désigné préférentiellement ce qui était indigène et dans une certaine mesure inassimilé, voire inassimilable. On peut, à titre de comparaison, suivre l'acception particulière du qualificatif « chleuh » dans l'argot militaire puis populaire français entre 1920 et 1945 : cet ethnique sud-marocain finissant, à la suite de glissements sémantiques, par 
désigner les soldats allemands contre lesquels se battirent d'ailleurs de vrais Chleuhs enrôlés dans l'Armée d'Afrique.

91 La cause est entendue, les Mauri Barbari de l'Antiquité tardive seront ceux que les Arabes de la Conquête appelleront Berbères en les distinguant soigneusement des Rom et des Afariq des villes et des campagnes romanisées. Aux siècles de la domination romaine, de même qu'il existe donc des tribus maures restées en dehors de la Romanitas, il est aussi des divinités aux caractères fuyants qui n'ont pas trouvé place dans le panthéon officiel. Les unes et les autres sont «maures». La foule de ces dieux indigènes reste innomée, d'où l'usage commode d'un collectif, qui est en même temps un qualificatif, lorsque l'on veut se les concilier.

Ainsi les Dii Mauri et ceux que, faute de mieux, nous avons appelés les dieux locaux, sont les mêmes divinités, mais les premiers sont invoqués, presque exclusivement $(81,25 \%)$ par des militaires et des fonctionnaires impériaux, les seconds sont honorés localement par de simples particuliers ou des magistrats municipaux. Les dieux sont les mêmes, ce sont les dédicants qui changent.

\section{BIBLIOGRAPHIE}

BENABOU M., La résistance romaine à la romanisation, Paris, F. Maspéro, pp. 309-330

CAMPS G, «L'inscription de Béja et le problème des Dii Mauri », Rev. afric., t. XCVIII, 1954, pp. 235-260.

CAMPS G., « Les Bavares, peuples de Maurétanie césarienne », Rev. afric, t. XCIX, 1955, pp. 241-288.

CHAKER S., « Onomastique berbère ancienne (Antiquité/Moyen Age) : rupture et continuité », B.C.T.H., nlle sér. fase. 19 B,1985, pp. 483-497.

CHERBONEAU M., « Excursion dans les ruines de Mila, Sufevar, Sila et Sigus pendant l'été de 1863 », Ree. de la Soc. archéol. de Constantine, t. 12, 1868, pp. 393-471 (p. 455).

ELMAYER A.F., « The libyan god Gurzil in a neo-punic inscription from Tripolitania », Libyan studies, XIII, 1982, pp. 49-50.

ENNAIFER M., « Note sur trois acquisitions des Musées archéologiques », Africa, t. VII-VIII, 1982, pp. 157-160.

FENTRESS E., « Dii Mauri and Dii Patrii », Latomus, 1978, pp. 505-516.

FÉVRIER J., « A propos de Ba'al Addir », Semitica, II, pp. 21-28.

FÉVRIER J., « Sur quelques noms puniques et Libyques », B.C.T.H., 1949, pp. V-VIII.

FÉVRIER P.-A., « Le Maure ambigu ou les pièges du discours », B.C.T.H., fase. 19 B, 1983 (1985), pp. 291-306.

FERRON J., « Restauration de l'autel et gravure d'une image sacrée dans un sanctuaire sahélien de B'al Addir », REPPAL, III, 1987, pp. 193-227. 
GALAND L., « Les Quinquegentiens », Bull, d'Archéol. algér., t. IV, 1970, pp. 297-299.

GSELL S., Histoire ancienne de l'Afrique du Nord, t. VI, Paris, 1928.

JOLEAUD L., « Gravures rupestres et rites de l'eau en Afrique du Nord », Joum. de la Soc. des African., t. III, 1933, pp. 197-282.

LASSERE J.M., Ubique populus. Peuplement et mouvements de population dans l'Afrique romaine, Paris, CNRS, Études d'Antiquités africaines, 1977.

LE GLAY M., « Le serpent dans les cultes africains », Latomus, 1957, pp. 338-353.

LE GLAY M., Saturne africain. Histoire. Paris, de Boccard, 1966.

LENOIR M., « Aulisua, dieu maure de la fécondité. III Convegno di studio su "l'Africa romana" ». Sassari, 13-15 décembre 1985.

MERLIN A., Divinités indigènes sur un bas-relief romain de la Tunisie, CRAIBL, 1947, pp. 355-371.

PEYRAS J., Le Tell nord-est tunisien dans l'Antiquité. Essai de monographie régionale, Paris, CNRS, Études d'Antiquité africaines, 1991.

PfLAUM H.G. « Remarques sur l'onomastique du Castellum Celtianum, Camuntina », III, 1956,

pp. 126-151.

REBUfFAT R. et MARICHAL R., « Les ostraca de Bu Njem ». Rev. des Et. latines, t. LI, 1973, pp. 281-286.

REBUFFAT (R.), « Ara cerei », M.E.F.R.A., t. XCIV, 1982, p. 911-919.

TOUTAIN (J.), Les Cultes païens dans l'Empire romain : cultes africains, 1905-1917,t. III, p. 59.

\section{INDEX}

Mots-clés : Antiquité, Libyque, Mythologie, Onomastique, Religion 